\title{
Anastomosis in the absence of a suprahyoid release following circumferential sleeve resection is feasible in differentiated thyroid carcinoma patients with tracheal invasion
}

\author{
WANJUN CHEN, SHUJUAN ZOU, LIANG WANG, CHANGHUA WU, \\ ZHIQI WANG, KE LI and SHUGUANG ZHANG \\ Department of Head and Neck Surgery, Shandong Cancer Hospital Affiliated to Shandong University, \\ Shandong Academy of Medical Sciences, Jinan, Shandong 250117, P.R. China \\ Received February 13, 2016; Accepted January 19, 2017
}

DOI: $10.3892 / \mathrm{ol} .2017 .6568$

\begin{abstract}
Invasion of the trachea is observed in 6\% of patients with differentiated thyroid carcinoma (DTC), and surgery is accepted as the treatment of choice. However, surgical procedures can be challenging and are associated with various risks. The authors of the present study performed a retrospective study of patients with DTC and tumor invasion of the trachea. Outcomes from patients that received circumferential sleeve resection (CSR) of the trachea followed by anastomosis in the absence of suprahyoid release ( $n=21$; CSR group) and patients that underwent tangential resections $[n=103$; tangential 'shave' resection (TSR) group) were analyzed. In the CSR group, 4 to 8 tracheal rings were circumferentially resected. All patients underwent end-to-end anastomosis in the absence of suprahyoid release following CSR, and 7 patients developed cancer metastasis following surgery. With the exception of 2 patients that succumbed to disease, the remaining patients in the CSR group survived without cancer recurrence. In the TSR group, all of the patients experienced cancer recurrence within five years post-surgery. A total of 61 patients developed metastases in the three years following surgery, and 71 patients succumbed to cancer metastasis within five years. The survival rate of the CSR group was significantly increased compared with the TSR group. The results of the present study suggest that in DTC patients with defects involving up to 8 tracheal rings, it may be appropriate to perform anastomosis without suprahyoid
\end{abstract}

Correspondence to: Dr Wanjun Chen, Department of Head and Neck Surgery, Shandong Cancer Hospital Affiliated to Shandong University, Shandong Academy of Medical Sciences, 440 Jiyan Road, Jinan, Shandong 250117, P.R. China

E-mail:wjchen2003@126.com

Abbreviations: DTC, differentiated thyroid carcinoma; CSR, circumferential sleeve resection; TSR, tangential 'shave' resection

Key words: thyroid, carcinoma, trachea, surgery, anastomosis, circumferential sleeve resection, suprahyoid release tissue release as it is associated with a reduced incidence of perioperative morbidity.

\section{Introduction}

The prognosis for patients with differentiated thyroid carcinoma (DTC) is very good, with the postoperative 10-year survival rate close to $90 \%$ (1). According to a recent study, there was an association between the size of the primary tumor and overall survival: Event-free and overall survival were significantly associated with increasing tumor size (2). Furthermore, a pT4a category and a tumor diameter $>2 \mathrm{~cm}$ remained independent predictors of survival (3). Tumor invasion of the larynx or trachea is observed in $\sim 6 \%$ of DTC cases (4), and invasion is an independent predictor of mortality. Furthermore, airway obstruction is the cause of mortality in $50 \%$ of patients with thyroid carcinoma (5). Currently, surgery is the most effective treatment for DTCs as the tumors are commonly insensitive to radiotherapy and chemotherapy (6).

For patients with advanced invasive thyroid carcinoma, the extent of surgical resection remains controversial (7). For superficial airway invasion, certain surgeons prefer to perform tangential excision, and therefore cancer recurrence may be common (7). For patients with transmural or laryngotracheal DTC invasion, circumferential sleeve resection (CSR) of the trachea with primary end-to-end anastomosis may be the optimal surgical choice, as complete resection of the tumor provides the longest overall survival and the greatest palliative effect (7). The advantages of this approach include the immediate reconstruction of the airway and preservation of a moist environment within the trachea (8). Although some patients with thyroid carcinoma are referred with tracheal or laryngotracheal invasions, indications for the use of salvage procedure have decreased over time. Tracheal resection is an appropriate intervention for DTC and provides good overall outcomes (9). However, this treatment strategy can be difficult and is associated with various risks. The present investigation is a retrospective study of patients with DTC and tracheal invasion. Outcomes from patients $(n=21)$ that received CSR of the trachea followed by anastomosis in the absence of suprahyoid release (CSR group) and patients $(n=103)$ that underwent 
tangential resections [tangential 'shave' resection (TSR) group] were analyzed.

\section{Materials and methods}

Patient characteristics and diagnosis. Between January 2004 and July 2014, patients with thyroid carcinomas $(n=156)$ invading the trachea and/or larynx were treated at the Department of Head and Neck Surgery of Shandong Cancer Hospital affiliated to Shandong University (Jinan, China). Ethical approval for the study was gained from the institutional review board at the Shandong Cancer Hospital affiliated to Shandong University. Written informed consent was obtained from the patients prior to obtaining follow-up information. Pathological specimens were assessed for resection margin status and histological characteristics by a single experienced pathologist. The tumor size was defined as the maximum tumor diameter on the original pathology report. To analyze the extent of invasion, the Shin staging system was used (10). Of the 156 patients, 32 (20.5\%) were excluded from further analysis due to refusal of surgery or the development of extensive local or advanced distant metastasis that precluded resection. TSR was performed in 103 patients (20 males and 83 females) with thyroid carcinomas invading the trachea. In the TSR group, the age of the patients ranged between 35 and 76 years (mean, 57 years). The common symptom of each patient was cervical mass, and voice changes were evident in 84 patients. Tracheal bleeding with hemoptysis occurred in 40 patients and 65 experienced grade $\geq$ II dyspnea, assessed as described previously (11). A total of 21 patients (5 males and 16 females) underwent tracheal reconstruction following CSR. In the CSR group, the age of the patients ranged between 35 and 72 years (mean, 60 years). The major presenting symptoms of each patient were the presence of a cervical mass and various degrees of respiratory difficulty. Of the 21 patients, 15 experienced grade $\geq$ II dyspnea. Tracheal bleeding with hemoptysis occurred in 9 patients and voice changes were evident in 18 patients.

The diagnosis of DTC tracheal invasion was established in 1 patient using fibre optic bronchoscopy on a preoperative biopsy specimen. For the remaining patients in the study, preoperative diagnosis was confirmed by endoscope and hemoptysis in 9 patients, and radiological examination was performed via $\mathrm{CT}$ scan in 16 patients. In the 5 remaining patients, tracheal invasion was suspected based on CT results, and biopsy specimens were taken during surgery to establish the diagnosis in the CSR group.

Surgical procedures. The surgical procedures required total or partial thyroidectomy, in conjunction with sleeve resection of the trachea and unilateral or bilateral neck dissection. All patients received general endotracheal anesthesia using an endotracheal tube, which was passed distal to the lower border of the tumor.

Following neck dissection and thyroidectomy, all patients received CSR of the trachea followed by end-to-end anastomosis. The trachea was dissected anteriorly and posteriorly, and subsequently mobilized in a step-by-step manner. Under direct vision, the trachea was circumferentially transected above and below the level of tumor invasion. A single layer of interrupted 4-0 prolene sutures were placed posteriorly through the membranous wall and subsequently anteriorly through the cartilaginous rings. Following suturing, the patient's head was placed in a flexed position. The anastomotic sutures were tied, ensuring the knots of the sutures were outside the tracheal lumen. To confirm the adequacy of the anastomosis, the anastomotic site was covered with saline solution, and the anesthesiologist released the balloon on the endotracheal tube to allow positive breathing pressure. If air bubbles were observed at the suture line, additional sutures were placed to secure a watertight closure. Suction drains were introduced, and the incision was closed in layers using fine surgical silk sutures for platysma and skin. To prevent inadvertent extension of the neck, the head was maintained in a flexed position for 2 weeks via two stitches placed in the submental crease and the upper thoracic skin.

In the TSR group $(n=103)$, following neck dissection, total thyroidectomy was performed in 20 patients $(19.4 \%)$ and partial thyroidectomy was performed in 83 patients $(80.6 \%)$. A 'shave' resection consisted of tangential excision of the tumor and thyroid gland occasionally associated by scraping the thinned-out tracheal wall. No patients underwent concomitant lateral excision of the full-thickness esophageal wall.

Postoperative therapy. Postoperative radiotherapy or radioactive iodine therapy was administered selectively to patients with incomplete resections or as preventative treatment. A recommended dose of 60 Gy to the neck and superior mediastinum was administered between 6 and 8 weeks after resection. In the present study, radioactive iodine was administered postoperatively to 12 patients in the CSR group. In the TSR group, 30 patients received postoperative radioactive iodine and 73 patients received postoperative radiotherapy. Patients with thyroidectomies were administered with levothyroxine postoperatively. For the majority of patients, an initial starting dose of $100 \mu \mathrm{g} / \mathrm{day}$ levothyroxine sodium (Merck KGaA, Darmstadt, Germany) was recommended, with gradual increments in dose at intervals of between 6 and 8 weeks, as required. The levothyroxine sodium dose was generally adjusted in increments of between 12.5 and $25 \mu \mathrm{g}$ increments until the serum TSH was $<0.5 \mu \mathrm{IU} / \mathrm{ml}$.

Statistical analysis. The outcomes of the different treatment strategies were analyzed using SPSS software (version 16; SPSS, Inc., Chicago, IL, USA). $\chi^{2}$ and Student's t-tests were performed to determine statistical differences and compare the data between the CSR and TSR group. The Kaplan-Meier method with log-rank testing was conducted to estimate the postoperative survival and compare the different groups. $\mathrm{P}<0.05$ was considered to indicate a statistically significant difference.

\section{Results}

Patient characteristics. The characteristics of the patients in the CSR group are presented in Table I and postoperative data are displayed in Table II. There were no significant differences in age, sex, common symptoms, tumor size, local lymph node metastasis, invasion of the recurrent laryngeal nerve or tumor type between the CSR and TSR groups (Tables III and IV). 


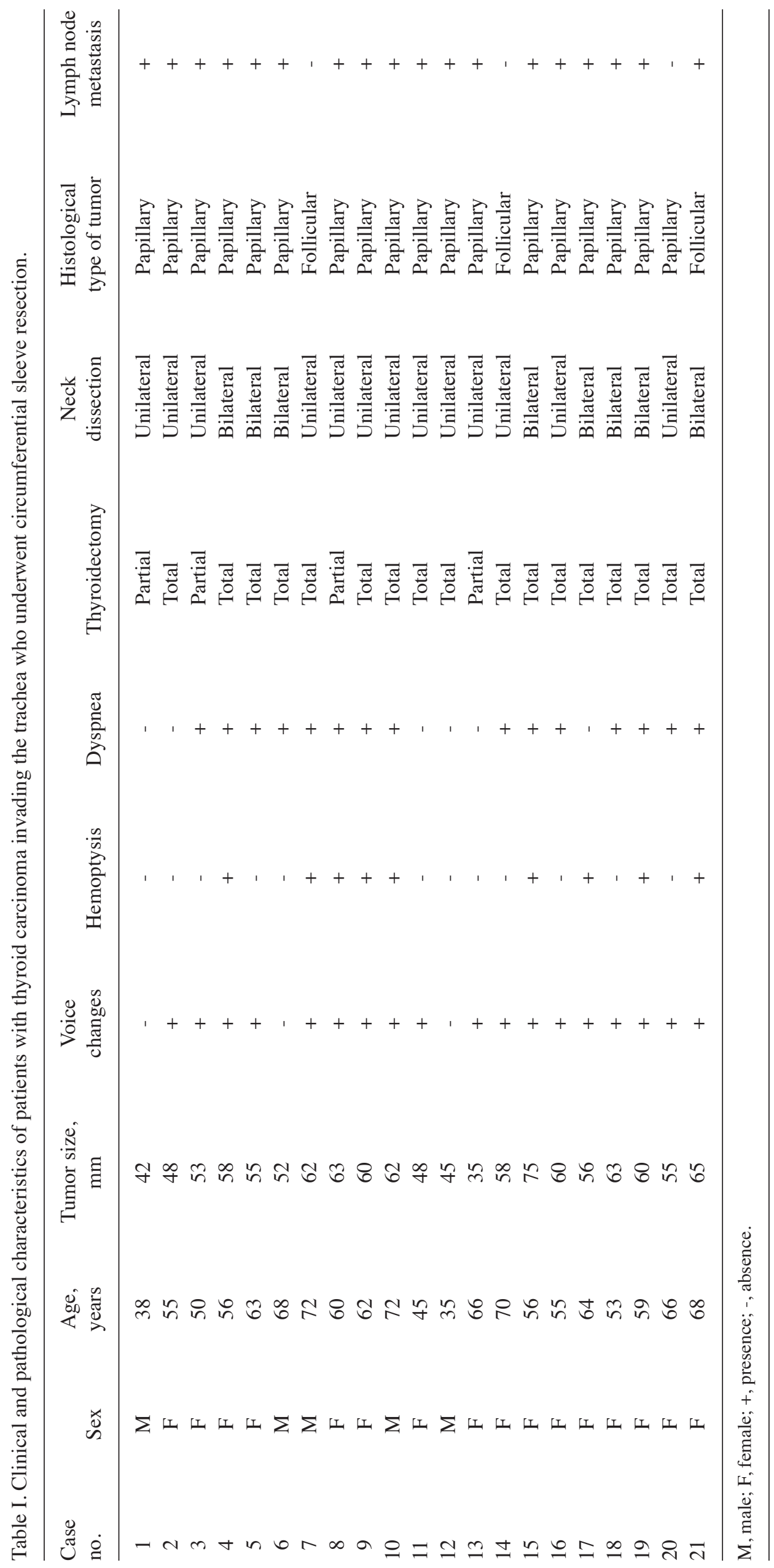




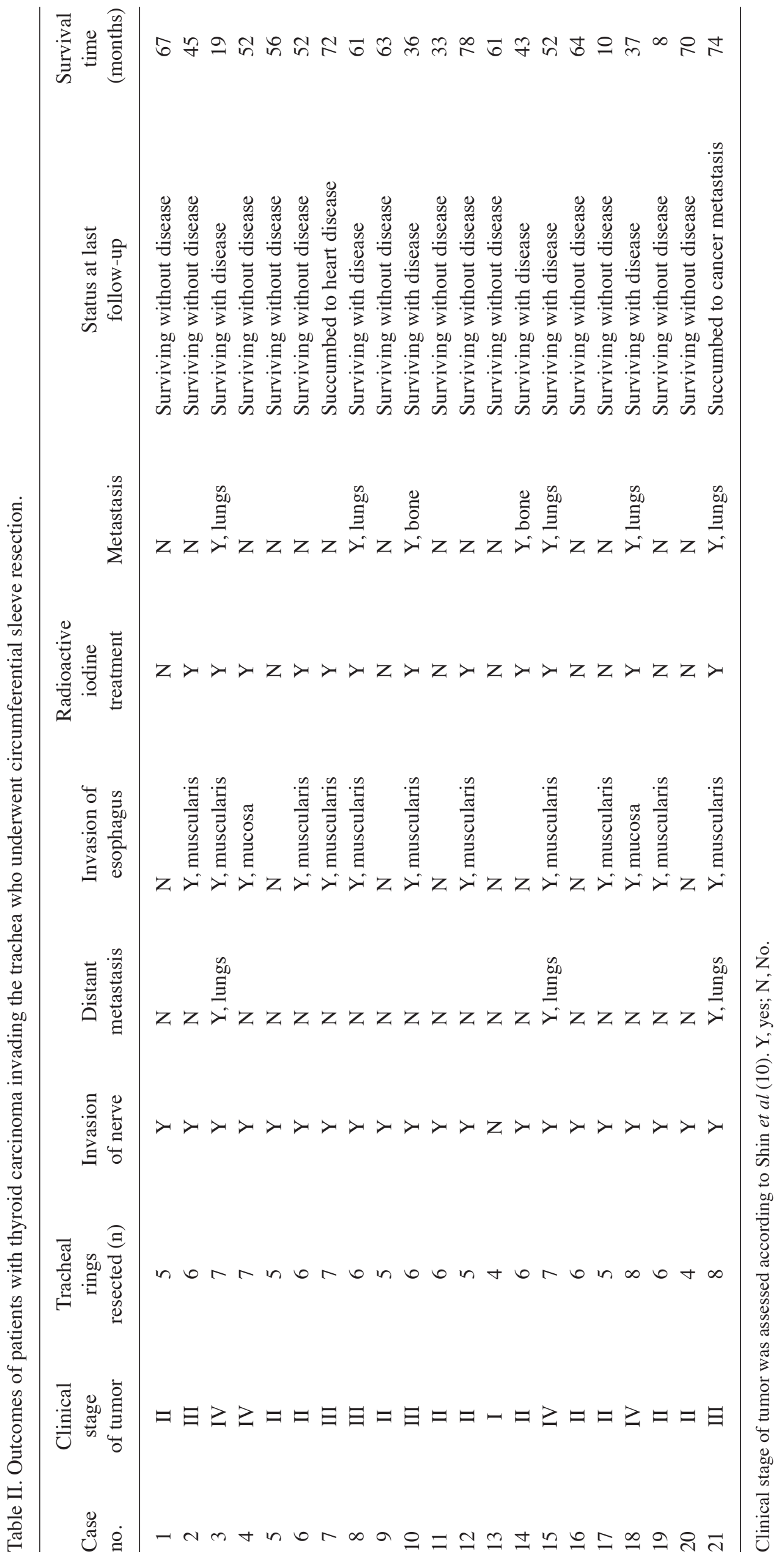


Table III. Characteristics of patients with thyroid carcinoma invading the trachea who underwent circumferential sleeve resection $(n=21)$ and tangential 'shave' resection $(n=103)$.

\begin{tabular}{|c|c|c|c|c|}
\hline Characteristic & $\begin{array}{l}\text { Circumferential } \\
\text { sleeve resection }\end{array}$ & $\begin{array}{c}\text { Tangential } \\
\text { 'shave' resection }\end{array}$ & P-value & $\chi^{2}$ \\
\hline Age, years ${ }^{a}$ & $60(35-72)$ & $57(35-76)$ & 0.457 & \\
\hline \multicolumn{5}{|l|}{ Sex, n (\%) } \\
\hline Male & $5(23.8)$ & $20(19.4)$ & 0.648 & 0.209 \\
\hline Female & $16(76.2)$ & $83(80.6)$ & & \\
\hline \multicolumn{5}{|c|}{ Symptoms, n (\%) } \\
\hline Mass & $21(100.0)$ & $103(100.0)$ & & \\
\hline Voice changes & $18(85.7)$ & $84(81.6)$ & 0.989 & 0.125 \\
\hline Hemoptysis & $9(42.9)$ & $40(38.8)$ & & \\
\hline Dyspnea & $15(71.4)$ & $65(63.1)$ & & \\
\hline \multicolumn{5}{|c|}{ Thyroidectomy, n (\%) } \\
\hline Total & $17(81)$ & $20(19.4)$ & $<0.0001$ & 31.55 \\
\hline Partial & $4(19)$ & $83(80.6)$ & & \\
\hline \multicolumn{5}{|c|}{ Neck dissection, n (\%) } \\
\hline Unilateral & $13(61.9)$ & $86(83.5)$ & 0.025 & 5.052 \\
\hline Bilateral & $8(38.1)$ & $17(16.5)$ & & \\
\hline
\end{tabular}

${ }^{a}$ Data are presented as the median and range. The dichotomous variables were compared using $\chi^{2}$ or Fisher's exact tests, as appropriate. All $\mathrm{P}$-values were two-sided.

Table IV. Outcomes of patients with thyroid carcinoma invading the trachea who underwent circumferential sleeve resection $(n=21)$ and tangential 'shave' resection $(n=103)$.

\begin{tabular}{|c|c|c|c|c|c|}
\hline Characteristic & $\begin{array}{l}\text { Circumferential } \\
\text { sleeve resection }\end{array}$ & $\begin{array}{c}\text { Tangential } \\
\text { 'shave' resection }\end{array}$ & P-value & $\chi^{2}$ & $\mathrm{t}$ \\
\hline \multicolumn{6}{|l|}{ Histological tumor type, n (\%) } \\
\hline Papillary & $18(85.7)$ & $82(79.6)$ & 0.519 & 0.416 & \\
\hline Follicular & $3(14.3)$ & $21(20.4)$ & & & \\
\hline Size of tumor, $\mathrm{mm}^{\mathrm{a}}$ & $55.96 \pm 8.89$ & $54.42 \pm 8.90$ & 0.473 & & 0.720 \\
\hline \multicolumn{6}{|l|}{ Lymph node metastasis, n (\%) } \\
\hline Yes & $18(85.7)$ & $94(91.3)$ & 0.433 & 0.614 & \\
\hline No & $3(14.3)$ & $9(8.7)$ & & & \\
\hline \multicolumn{6}{|l|}{$\begin{array}{l}\text { Invasion to the recurrent } \\
\text { laryngeal nerve, } \mathrm{n}(\%)\end{array}$} \\
\hline Yes & $20(95.2)$ & $95(92.2)$ & 0.629 & 0.234 & \\
\hline No & $1(4.8)$ & $8(7.8)$ & & & \\
\hline \multicolumn{6}{|l|}{ Complications, n (\%) } \\
\hline Air leak & $5(23.8)$ & $2(1.9)$ & & & \\
\hline Temporary hypoparathyroidism & $9(42.9)$ & $30(29.1)$ & 0.018 & 10.068 & \\
\hline Temporary dysphagia & $11(52.4)$ & $21(20.4)$ & & & \\
\hline Lymphatic fistulas & $4(19.0)$ & $23(22.3)$ & & & \\
\hline
\end{tabular}

a'Data are presented as the mean \pm standard deviation. Continuous variables were compared using Student's t-test, whereas dichotomous variables were compared using $\chi^{2}$ or Fisher's exact tests, as appropriate. All P-values were two-sided.

Anastomosis of tracheal defects involving up to 8 rings following tumor resection. A representative image from the radiological examination performed via CT scan in 16 patients is presented in Fig. 1. All patients in the CSR group $(n=21)$ 


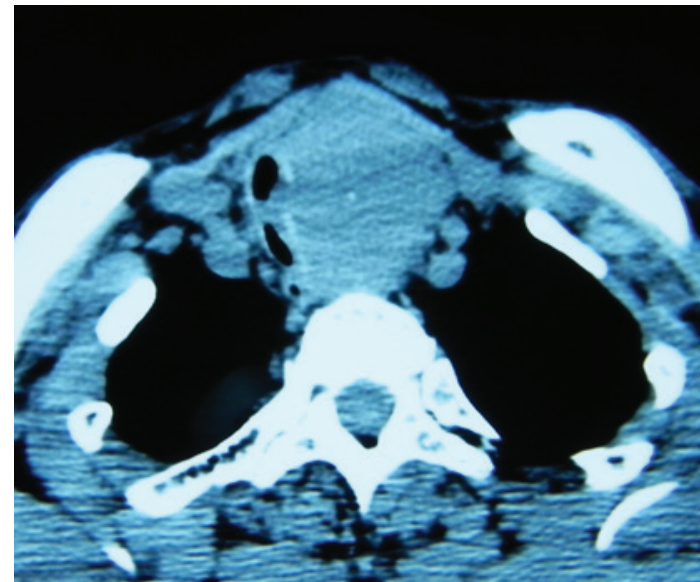

Figure 1. CT scan showing thyroid tumor with invasion of the cervical trachea.

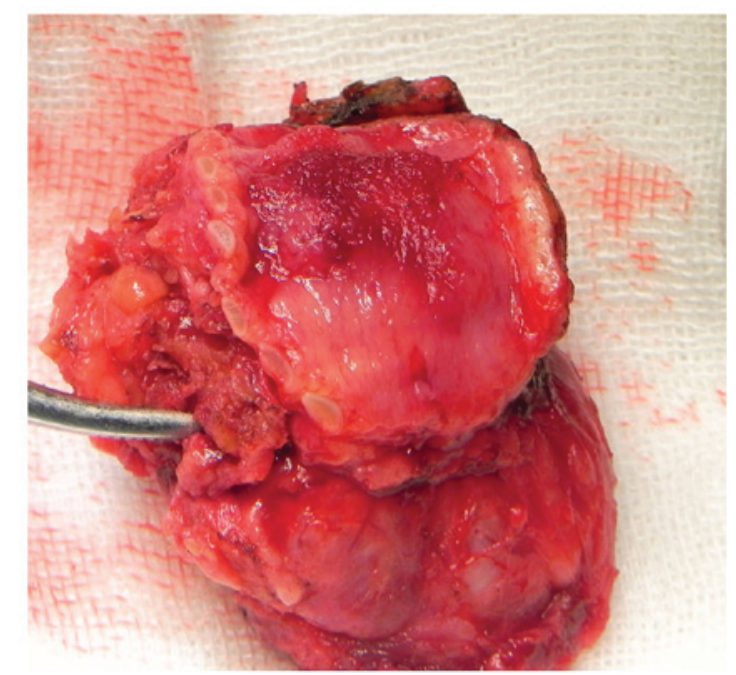

Figure 2. Papillary thyroid carcinoma in resected trachea segment.

had stage pT4a tumors, classified according to the American Joint Committee on Cancer (AJCC) (12). Total thyroidectomy was performed in 17 cases $(81 \%)$ and partial thyroidectomy in 4 cases (19\%). Resection of the recurrent nerve on the affected side was performed in 20 patients $(95.2 \%)$ and $2(9.5 \%)$ underwent concomitant lateral excision of the full-thickness esophageal wall with primary closure. The extent of the resection ranged from 4 to 8 tracheal cartilage rings. In 2 patients, 4 tracheal rings were resected. A total of 5 tracheal rings were resected in 5 patients, 6 in 8 patients, 7 in 4 patients and 8 in 2 patients (Figs. 2 and 3 ).

In the TSR group $(n=103)$, total thyroidectomy was performed in 20 patients $(19.4 \%)$ and partial thyroidectomy in 83 patients $(80.6 \%)$. No patients underwent concomitant lateral excision of the full-thickness esophageal wall. Although all patients had stage pT4a tumors (12), which were similar to that of the CSR group, they received TSR. TSR was performed because certain patients had developed extensive local disease that precluded primary anastomosis. Others had developed advanced distant metastasis or cardiovascular diseases; therefore, they were unable to tolerate radical surgery. Additionally, certain patients received TSR as they refused radical surgery or the surgeons decided that mediastinal tracheostomy must be performed during operative exploration. In order to reduce complications and improve the quality of life, the majority of these patients in the TSR group (80.6\%) underwent partial thyroidectomy rather than total thyroidectomy.

No occurrence of serious complications following tracheal anastomosis without suprahyoid release. In the CSR group $(\mathrm{n}=21)$, no serious complications occurred following CSR of the trachea. In 5 (23.8\%) patients, air leaks were observed on day 1 post-surgery. However, bronchoscopy revealed no wound dehiscence, and these leaks resolved spontaneously. Temporary hypoparathyroidism occurred in 9 patients following total thyroidectomy $(52.9 \%)$, and permanent hypoparathyroidism was not observed in any of the patients. Temporary postoperative dysphagia occurred in 11 patients in the CSR group $(52.4 \%)$. The surgical morbidity of the CSR group was marginally higher compared with the TSR group, and this was due to the wider surgical field and the more complicated nature of the procedure. However, the postsurgical complications were not serious and were resolved quickly. Notably, no patients required tracheostomy following surgery.

Similarly, no serious complications occurred in the patients of the TSR group $(n=103)$. The postoperative recovery was markedly improved compared with the CSR group. Temporary hypoparathyroidism occurred postoperatively in 37 patients $(35.9 \%)$, and temporary postoperative dysphagia occurred in 20 patients (19.4\%). Prophylactic tracheostomies were performed in 20 patients $(19.4 \%)$ to prevent postoperative complications and 11 patients $(10.7 \%)$ required permanent tracheostomy.

Complete resection of stage I to IV DTC with tracheal invasion by primary anastomosis without suprahyoid release. In the CSR group, the size of the primary thyroid tumor was $55.96 \pm 8.89 \mathrm{~mm}$ (mean \pm standard deviation; range, 35 to $75 \mathrm{~mm}$ ). Invasion into the recurrent laryngeal nerve was observed in 20 patients $(95.2 \%)$. A total of 4 to 8 tracheal rings (mean, 5.9 rings) were circumferentially resected during surgery, including a partial resection of the cricoid cartilage in 8 patients. The tracheal wall invasions were limited to the adventitia in 1 patient [Stage I; 4.8\%, (10)]. Tracheal invasions were also observed between the tracheal rings in 11 patients (Stage II; 53.4\%) and within the submucosal space in 5 patients (Stage III; 23.8\%). Additionally, protrusions into the tracheal lumen were detected in 4 patients (Stage IV; 19\%). Tumor tissues were absent in the specimen margins in all patients in the CSR group. Histological examination indicated that the tumors were papillary carcinomas in 18 patients $(85.7 \%)$ and follicular carcinomas in 3 patients $(14.3 \%)$. All patients underwent anastomosis without suprahyoid release following CSR of the trachea. No patients received tracheostomy prior to or following sleeve resection of the trachea.

In the TSR group, the primary thyroid tumor size was $54.42 \pm 8.90 \mathrm{~mm}$ (mean \pm standard deviation; range, 36 to $74 \mathrm{~mm}$ ). Invasion into the recurrent laryngeal nerve was observed in 95 patients $(92.2 \%)$. The grade of the tumors was not microscopically evaluated due to a lack of specimens from the TSR group. 


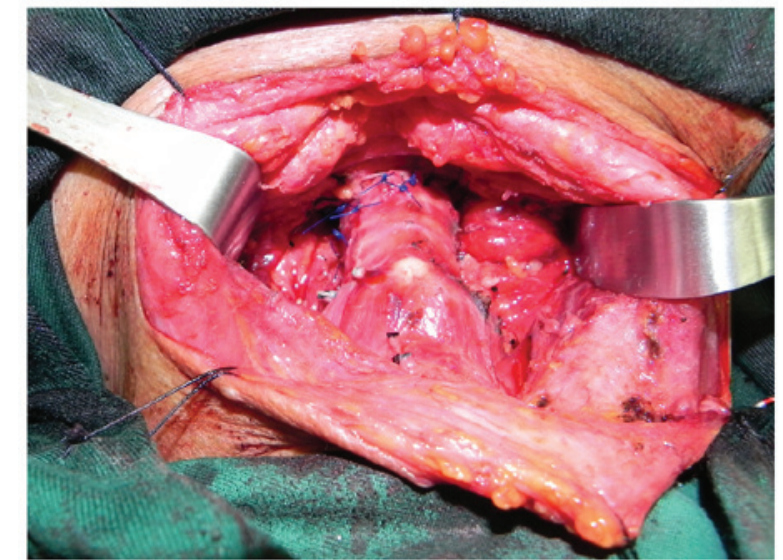

Figure 3. Reconstruction of trachea by end-to-end anastomosis following circumferential sleeve resection.

Good long-term outcomes following complete tumor resection and tracheal anastomosis in all DTC patients. The follow-up period for patients in the present study ranged from 1 to 10 years (mean, 5 years and 7 months). In the CSR group, radioactive iodine was administered postoperatively to 12 patients $(57.1 \%)$. In 3 patients, whole-body scans following treatment detected lung metastases, and these patients responded to therapy and are currently disease-free. To reduce cancer recurrence and prolong survival, radioactive iodine treatment was administered to 5 patients postoperatively. Radioactive iodine therapy was administered to 4 patients that had developed further pulmonary and/or bone metastases at 3 years post-surgery. There were no primary site recurrences in the patients in the CSR group. Selective neck dissection was performed on 1 patient due to contralateral lymph node metastasis 4 years post-surgery. At the end of the follow-up period, 1 patient had succumbed to distant metastasis 7 years post-surgery, and 1 patient succumbed to heart disease 6 years post-surgery. The remaining patients survived without cancer recurrence throughout the postoperative period. In summary, only 1 patient in the CSR group succumbed to disease associated with thyroid carcinoma, 13 patients were disease-free and 6 survived with disease.

In the TSR group, 56 of the 103 patients developed primary site recurrences $(54.4 \%)$ within 3 years post-surgery. The rate of cancer recurrence was significantly higher compared with the CSR group. All patients developed significant primary site recurrences within 5 years, and 61 developed further pulmonary and/or bone metastases within 3 years post-surgery (59.2\%). At the end of the follow-up period, 70 patients succumbed to distant metastases within 5 years (68\%).

Furthermore, the survival rate of patients in the CSR group that received complete resection was significantly higher compared with patients in the TSR group that underwent incomplete resection $\left(\chi^{2}=79.2, \mathrm{P}<0.0001\right.$; Fig. 4). Patients that underwent tracheal anastomosis without suprahyoid release following CSR exhibited improved long-term outcomes compared with the patients in the TSR group.

\section{Discussion}

In DTC, the extent of airway infiltration is an important determinant of outcome, as deep tracheal invasion and laryngeal

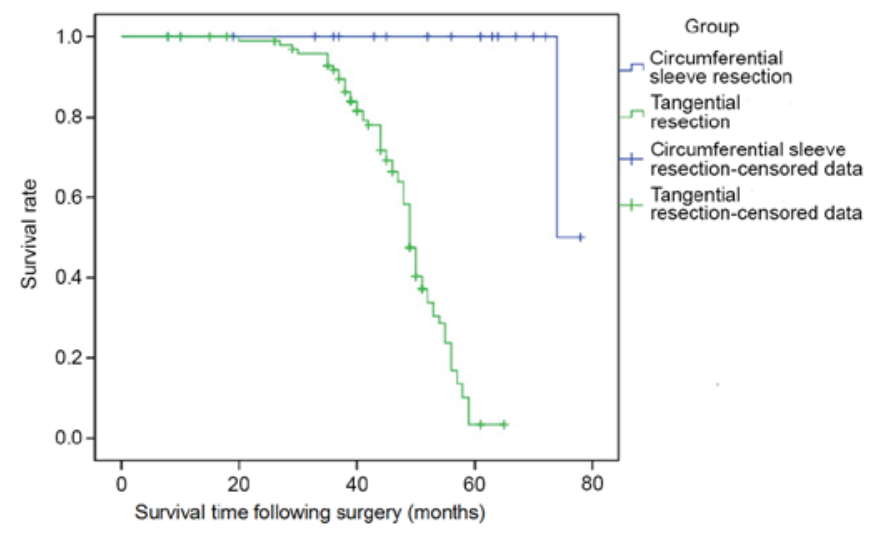

Figure 4. Postoperative patient survival times.

infiltration are correlated with poorer prognosis (13). Regarding surgical strategies, classification of aerodigestive tract invasion is based on a three-dimensional assessment to determine surgical resection options. Incomplete tangential wall resection may be performed if the invasion is superficial. By contrast, deep wall and intraluminal invasions require complete wall resection (14). In the report by McCarty et al (7), 6/35 patients with superficial tracheal adherence that received tracheal shaving exhibited local recurrence after 10 years (17\%). In the present study, the tumor margins were difficult to assess in the TSR group. Therefore, microscopic residual disease may have been left behind. Thus, the process of invasion could not be determined in these patients. Mossetti et al (15) suggested performing 'shave' resections with biopsies of the tracheal wall surface in the area of infiltration, and if the biopsies are positive, to proceed with a sleeve resection in a subsequent surgery. However, full-thickness resection of the tracheal or laryngotracheal wall is required to achieve complete tumor clearance (16). A previous study reported that survival was increased in patients with thyroid cancer that received airway-resection compared with a non-resected group (17). Furthermore, the study also demonstrated that malignant tissue that is not completely removed from the tracheal wall cannot be controlled with adjuvant treatment and thus may result in early recurrence (17). In the long-term follow-up of the present study, the authors observed that there was a high risk of recurrence following the shaving procedures, even when followed with postoperative radioactive iodine or radiotherapy. The rate of relapse in the patients who underwent TSR was $54.4 \%$ within 3 years and $100 \%$ within 5 years, demonstrating that the benefits of adjuvant therapy were very limited.

Airway complications, recurrent disease and salvage operations were associated with decreased survival. By contrast, airway resection following thyroidectomy and complete resection were associated with improved prognosis (1). Therefore, salvage resections should be performed only in selected cases (18). Even when distant metastases are also present, segmental airway resection may prolong survival, control disease and lead to increased long-term survival in certain patients (19). Only complete full-thickness airway resections are able to improve clinical outcomes in DTC patients with deep invasion of the trachea (20). The 5-year overall survival rate following resection with curative intent is $70 \%$, whereas it 
is $<40 \%$ in palliative treatment patients (8). Thus, resection is a useful surgical intervention to treat differentiated tumors and provides good overall outcome (8).

Circumferential sleeve resection for DTC with tracheal invasion is a challenging procedure. The length of the expected gap in the continuity of the windpipe depends on the longitudinal extent of tumor infiltration into the trachea and the length of the patient's neck. Thus, these lengths determine whether suprahyoid release, or combined cervical and mediastinal release is appropriate. Transient dysphagia often occurs following suprahyoid release and may lead to aspiration pneumonia (21). No patients in the CSR group in the present study underwent suprahyoid release, and dysphagia was not prolonged in the majority of patients, where the symptoms eased and disappeared gradually within a week. Additionally, no patients required monitoring in the intensive care unit for signs of complications. Therefore, in the view of the authors, suprahyoid release may not be required following anastomosis of the trachea when $<8$ tracheal rings have been resected. Consequently, the risks associated with the surgery and operative times may be reduced.

When deep tracheal invasion is limited to a small area and involves only up to a third of the tracheal circumference, a potential alternative surgical procedure is partial 'window' resection (22). This procedure is particularly useful for cases where the range of invasion is limited to half of the circumference of the tracheal wall or less. However, window resection has several drawbacks (23).

Anastomotic dehiscence is a fatal complication following surgical anastomosis. Therefore, laryngeal and distal tracheal release should be performed to prevent tension on the anastomosis. To release the larynx, it is necessary to resect the suprahyoid muscles from the hyoid bone, and the larynx descends by 2.0 to $2.5 \mathrm{~cm}$, which is sufficient for an end-to-end anastomosis (6). However, in the majority of patients, this procedure may cause moderate to severe dysphagia and may even lead to aspiration pneumonia when the condition is serious. In the present study, the suprahyoid muscles remained in the correct position and functioned normally. Temporary mild dysphagia occurred postoperatively in 11 patients (52.4\%), and no aspiration pneumonia was observed. Similarly, swallowing function recovered quickly. In the view of the authors, end-to-end anastomosis may not require suprahyoid muscle release when the extent of the resection ranges from 4 to 8 tracheal cartilage rings. To avoid inadvertent extension of the neck, the head must be maintained in a flexed position for 2 weeks using stitches. Maintaining this position is the most important step for avoiding anastomotic dehiscence.

In conclusion, in all of the patients in the present study, CSR with immediate reconstruction without suprahyoid release was the most favorable strategy, and excellent outcomes were achieved without serious complications. One advantage of this surgical approach is that the integrity of the natural airway is preserved without any reconstructive material, which enables the surgery to be completed in a single stage. Another advantage is that suprahyoid release may not be required. Therefore, the procedure is simplified, which shortens the operative time and decreases the rate of complications. Anastomotic reconstruction of the trachea following CSR without suprahyoid release creates a stable upper air passage and is associated with lower perioperative morbidity. However, the present study has several shortcomings that require improvement. For example, the follow-up times for patients with DTC were short. Therefore, accurate evaluations of the overall survival of the patients were difficult to obtain. Furthermore, the majority of patients who underwent CSR for invasive thyroid cancer $(90 \%)$ survived at the conclusion of the follow-up period. Therefore, the follow-up of these patients will be continued.

\section{References}

1. Gaissert HA, Honings J, Grillo HC, Donahue DM, Wain JC, Wright CD and Mathisen DJ: Segmental laryngotracheal and tracheal resection for invasive thyroid carcinoma. Ann Thorac Surg 83: 1952-1959, 2007.

2. Vrachimis A, Wenning C, Gerß J, Dralle H, Vaez Tabassi M, Schober O and Riemann B; MSDS study group: Not all DTC patients with $\mathrm{N}$ positive disease deserve the attribution 'high risk'. Contribution of the MSDS trial. J Surg Oncol 112: 9-14, 2015.

3. Kramer JA, Schmid KW, Dralle H, Dietlein M, Schicha H, Lerch H, Gerss J, Frankewitsch T, Schober O and Riemann B; MSDS study group: Primary tumour size is a prognostic parameter in patients suffering from differentiated thyroid carcinoma with extrathyroidal growth: Results of the MSDS trial. Eur J Endocrinol 163: 637-644, 2010.

4. Czaja JM and McCaffrey TV: The surgical management of laryngotracheal invasion by well-differentiated papillary thyroid carcinoma. Arch Otolaryngol Head Neck Surg 123: 484-490, 1997.

5. Ishihara T, Yamazaki S, Kobayashi K, Inoue H, Shimao F and Ito Kand Mimura T: Resection of the trachea infiltrated by thyroid carcinoma. Ann Surg 195: 496-500, 1982

6. Lin S, Huang H, Liu X, Li Q, Yang A, Zhang Q, Guo Z and Chen Y: Treatments for complications of tracheal sleeve resection for papillary thyroid carcinoma with tracheal invasion. Eur J Surg Onco 140: 176-181, 2014>

7. McCarty TM, Kuhn JA, Williams WL Jr,Ellenhorn JD, O'Brien JC, Preskitt JT, Lieberman ZH, Stephens J, Odom-Maryon T, Clarke KG and Wagman LD: Surgical management of thyroid cancer invading the airway. Ann Surg Oncol 4: 403-408, 1997.

8. Honings J, Stephen AE, Marres HA and Gaissert HA: The management of thyroid carcinoma invading the larynx or trachea. Laryngoscope 120: 682-689, 2010.

9. Avenia N, Vannucci J, Monacelli M, Lucchini R, Polistena A, Santoprete S, Potenza R, Andolfi M and Puma F: Thyroid cancer invading the airway: Diagnosis and management. Int J Surg 28 (Suppl 1): S75-S78, 2016.

10. Shin DH, Mark EJ, Suen HC and Grillo HC: Pathologic staging of papillary carcinoma of the thyroid with airway invasion based on the anatomic manner of extension to the trachea: A clinicopathologic study based on 22 patients who underwent thyroidectomy and airway resection. Hum Pathol 24: 866-870, 1993.

11. Tanigawa N, Sawada S, Okuda Y, Kobayashi M and Mishima K. Symptomatic improvement in dyspnea following tracheobronchial metallic stenting for malignant airway obstruction. Acta Radiol 41: 425-428, 2000.

12. Greene FL, Page DL, Fleming ID, et al: AJCC cancer staging. In: TNM Classification of Malignant Tumors. Sobin LH and Wittekind C (eds). 6th edition. Springer-Verlag, New York, NY, pp89-98, 2002.

13. Wada N, Nakayama H, Masudo Y, Suganuma N and Rino Y: Clinical outcome of different modes of resection in papillary thyroid carcinomas with laryngotracheal invasion. Langenbecks Arch Surg 391: 545-549, 2006.

14. Brauckhoff M: Classification of aerodigestive tract invasion from thyroid cancer: Langenbecks Arch Surg 399: 209-216, 2014.

15. Mossetti C, Palestini N, Bruna MC, Camandona M, Freddi M, Oliaro A and Gasparri G: Segmental tracheal resection for invasive differentiated thyroid carcinoma. Our experience in eight cases. Langenbecks Arch Surg 398: 1075-1082, 2013.

16. Brauckhoff M, Machens A, Thanh PN, Lorenz K, Schmeil A, Stratmann M, Sekulla C, Brauckhoff K and Dralle H: Impact of extent of resection for thyroid cancer invading the aerodigestive tract on surgical morbidity, local recurrence, and cancer-specific survival. Surgery 148: 1257-1266, 2010. 
17. Shadmehr MB, Farzanegan R, Zangi M, Mohammadzadeh A, Sheikhy K, Pejhan S, Daneshvar A and Abbasidezfouli A: Thyroid cancers with laryngotracheal invasion. Eur J Cardiothorac Surg 41: 635-640, 2012.

18. Brauckhoff $M$ and Dralle H: Extrathyroidal thyroid cancer: Results of tracheal shaving and tracheal resection. Chirurg 82 : 134-140, 2011 (In German).

19. Musholt TJ, Musholt PB, Behrend M, Raab R, Scheumann GF and Klempnauer J: Invasive differentiated thyroid carcinoma: Tracheal resection and reconstruction procedures in the hands of the endocrine surgeon. Surgery 126: 1078-1087, 1999.

20. Honings J, Stephen AE, Marres HA and Gaissert HA: The management of thyroid carcinoma invading the larynx or trachea. Laryngoscope 120: 682-689, 2010.
21. Sywak M, Pasieka JL, McFadden S, Gelfand G, Terrell J and Dort J: Functional results and quality of life after tracheal resection for locally invasive thyroid cancer. Am J Surg 185: 462-467, 2003.

22. Chernichenko N and Shaha AR: Role of tracheal resection in thyroid cancer. Curr Opin Oncol 24: 29-34, 2012

23. Ebihara M, Kishimoto S, Hayashi R, Miyazaki M, Shinozaki T, Daiko H, Saikawa M, Sakuraba M and Miyamoto S: Window resection of the trachea and secondary reconstruction for invasion by differentiated thyroid carcinoma. Auris Nasus Larynx 38: 271-275, 2011. 